Pesq. Vet. Bras. 35(2):165-168, fevereiro 2015 DOI: 10.1590/S0100-736X2015000200012

\title{
Aspectos anatomopatológicos do parasitismo por nematódeos da superfamília Filarioidea em capivaras (Hydrochoerus hydrochaeris) de vida livre no Centro-Oeste brasileiro ${ }^{1}$
}

\author{
Lorena F. Silva ${ }^{2}$, Janildo L. Reis Jr², Cláudio H.G. Barbosa ${ }^{2}$, Chris H. Gardiner ${ }^{3}$ \\ e Fabiano J.F. de Sant'Ana ${ }^{2 *}$
}

\begin{abstract}
Silva L.F., Reis Jr J.L., Barbosa C.H.G., Gardiner C.H. \& Sant'Ana F.J.F. 2015. [Anatomic pathology aspects of the parasitism by Cruorifilaria tuberocauda in capybaras (Hydrochoerus hydrochaeris) in Midwestern Brazil.] Aspectos anatomopatológicos do parasitismo por nematódeos da superfamília Filarioidea em capivaras (Hydrochoerus hydrochaeris) de vida livre no Centro-Oeste brasileiro. Pesquisa Veterinária Brasileira 35(2):165-168. Laboratório de Patologia Veterinária, Universidade de Brasília, Brasília, DF, 70910-900, Brazil. E-mail: santanafjf@yahoo.com

This report describes two cases of parasitism by Cruorifilaria tuberocauda in wild capybaras (Hydrochoerus hydrochaeris) from Distrito Federal, Brazil. Grossly, there was marked thickening of vessels wall of the cortical and corticomedullary regions of both kidneys. Microscopically, there was severe proliferative and granulomatous arteritis associated with intralesional filarids, consistent with Cruorifilaria tuberocauda. For the first time this filarid is reported parasitizing capybaras in Distrito Federal, Brazil.
\end{abstract}

INDEX TERMS: Cruorifilaria tuberocauda, capybara, Hydrochoerus hydrochaeris, kidney pathology, filarids.

RESUMO.- São descritos dois casos de parasitismo por Cruorifilaria tuberocauda em capivaras de vida livre no Distrito Federal, Brasil. Macroscopicamente, observou-se nas superfícies de corte dos rins espessamento acentuado de vasos das regiões cortical e córtico-medular. Microscopicamente, havia arterite proliferativa e granulomatosa acentuada associada a filarídeos intralesionais consistentes com Cruorifilaria tuberocauda. Esse é o primeiro relato do parasitismo por esse filarídeo em capivaras no Distrito Federal.

TERMOS DE INDEXAÇÃO: Cruorifilaria tuberocauda, capivara, Hydrochoerus hydrochaeris, patologia renal, filarídeos.

\section{INTRODUÇÃO}

A capivara, Hydrochoerus hydrochaeris, é o maior roedor do mundo, com indivíduos adultos variando de peso, entre 30 e $70 \mathrm{~kg}$ (Alho \& Rondon 1987). É um herbívoro semiaquá-

\footnotetext{
${ }^{1}$ Recebido em 29 de julho de 2014.

Aceito para publicação em 17 de dezembro de 2014

${ }^{2}$ Laboratório de Patologia Veterinária (LPV), Universidade de Brasília (UnB), Campus Universitário Darcy Ribeiro, L4 Norte, Asa Norte, Brasília, DF, 70910-900, Brasil.*Autor para correspondência: santanafjf@yahoo.com

${ }^{3}$ Veterinary Pathology Service, Joint Pathology Center, 606 Stephen Sitter Ave., Silver Spring, MD 20910-1290, USA.
}

tico cuja área de ocorrência se estende por grande parte da América do Sul, habitando bacias hidrográficas do Panamá em direção ao sul do Uruguai e norte da Argentina (Alho \& Rondon 1987). No Brasil, com exceção da região Nordeste, esta espécie está largamente distribuída, com destaque para a região do pantanal mato-grossense (Costa et al. 2002). Em populações naturais, doenças parasitárias não constituem a principal causa de morte de capivaras, mas em locais com baixa pressão de caça e poucos predadores naturais, esse problema assume maior importância (Corriale et al. 2011).

Cruorifilaria tuberocauda (Nematoda: Filarioidea) é um nematódeo filarídeo que parasita o rim, pulmão e coração de capivaras (Hydrochoerus hydrochaeris) de vida livre. A ocorrência desse parasito já foi descrita em países sul-americanos, como a Colômbia (Rodriguez et al. 1975, Eberhard et al. 1976, Morales et al. 1978) e Venezuela (Ojasti 1973, Campo-Aesen \& Planas-Giron 1986). C. tuberocauda já foi registrada nos estados brasileiros de Mato Grosso do Sul (Costa \& Catto 1994, Nascimento et al. 2000) e Minas Gerais (Vieira et al. 2006).

No Brasil, descrições desse nematódeo parasitando capivaras têm sido realizadas (Costa \& Catto 1994, Vieira et al. 2006) e, no entanto, em apenas um trabalho, as altera- 
ções morfológicas causadas por essa parasitose foram descritas (Nascimento et al. 2000).

O ciclo de vida de C. tuberocauda é pouco conhecido (Morales et al. 1978). Morfologicamente, os filarídeos adultos são robustos, afilados em ambas as extremidades e as fêmeas e os machos medem, em média, aproximadamente 42 e $27 \mathrm{~mm}$ de comprimento, respectivamente (Eberhard et al. 1976).

As lesões causadas pelos adultos de C. tuberocauda ocorrem principalmente nos vasos sanguíneos e consistem de inflamação granulomatosa, algumas vezes com morte do parasita no centro da lesão e mineralização. Ocasionalmente, microfilárias podem ser encontradas em glomérulos renais e lesões pulmonares (Morales et al. 1978).

Este trabalho objetiva descrever as características anatomopatológicas de dois casos de parasitismo por filarídeos consistentes com Cruorifilaria tuberocauda em capivaras de vida livre no Distrito Federal, Centro-Oeste brasileiro, e caracterizar os aspectos histopatológicos das lesões causadas por este filarídeo.

\section{MATERIAL E MÉTODOS}

Duas capivaras adultas ( $\mathrm{C} 1$ e C2), fêmeas, de vida livre, encon-

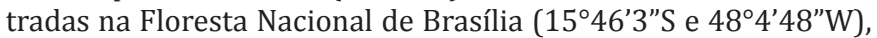
foram encaminhadas pelo Batalhão de Polícia Militar Ambiental (C1) e pelo Instituto Brasileiro do Meio Ambiente e dos Recursos Naturais Renováveis (IBAMA) (C2) para o Hospital Veterinário (HVet) da Universidade de Brasília (UnB), em março de 2014. 0 animal C1 apresentou histórico de cegueira bilateral com úlcera de córnea unilateral e foi eutanasiado com overdose de fenobarbital, diante do prognóstico desfavorável, uma vez que a reintrodução em vida livre de um animal silvestre e cego foi considerada inadequada. A capivara C2 estava apática, fraca, magra, em decúbito ventral e morreu naturalmente no dia seguinte da chegada ao HVet-UnB. Os dois animais foram submetidos à necropsia no Laboratório de Patologia Veterinária (LPV) da UnB, onde foram coletadas amostras de vários órgãos, que foram fixadas em formol a 10\%, desidratadas em álcool absoluto, diafanizadas em xilol e emblocadas em parafina. Foram realizados cortes de $4 \mu \mathrm{m}$ em micrótomo de rotação com posterior coloração pela hematoxilina-eosina (HE).

\section{RESULTADOS}

Na necropsia da capivara C1, foram observados carrapatos na pele. No baço, detectou-se esplenomegalia moderada com hiperplasia de polpa branca. As superfícies de corte dos rins revelaram espessamento moderado a acentuado de muitos vasos localizados na cortical e, principalmente, na junção córtico-medular (Fig.1). Adicionalmente, foram observadas, nessas últimas regiões, áreas hemorrágicas mal delimitadas de aproximadamente $2 \mathrm{~cm}$ de diâmetro. As córneas estavam moderadamente opacas e esbranquiçadas a levemente esverdeadas. A capivara C2 apresentou úlcera profunda na face lateral do membro pélvico esquerdo com numerosas larvas de moscas (miíases), dois abscessos pulmonares e as mesmas lesões renais detectadas no animal C1. Os demais órgãos não apresentaram alterações significativas nos dois animais.

Microscopicamente, nos dois animais, as artérias renais afetadas, principalmente as interlobares e interlobulares,

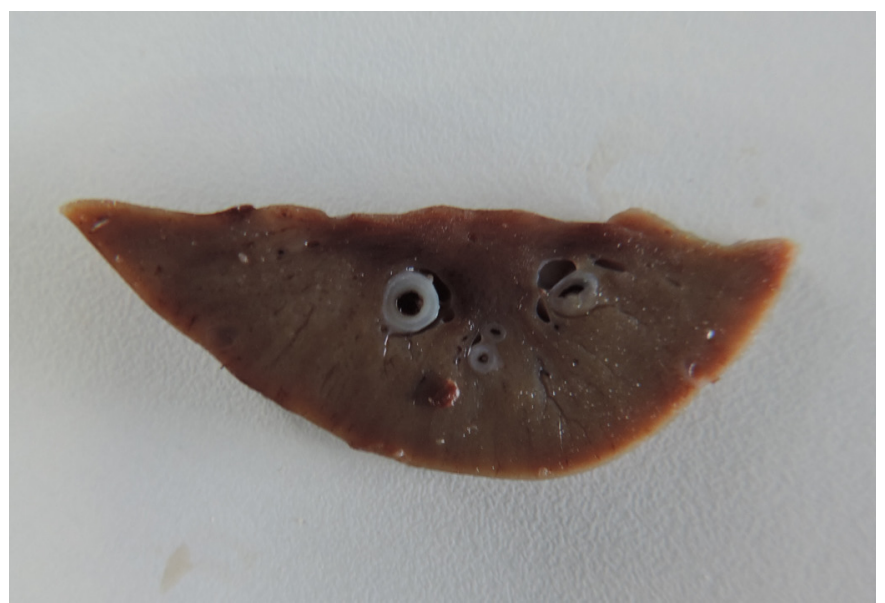

Fig.1. Superfície de corte de fragmento de rim fixado evidenciando espessamento acentuado de vasos localizados nas regiões cortical e córtico-medular (Capivara C1).

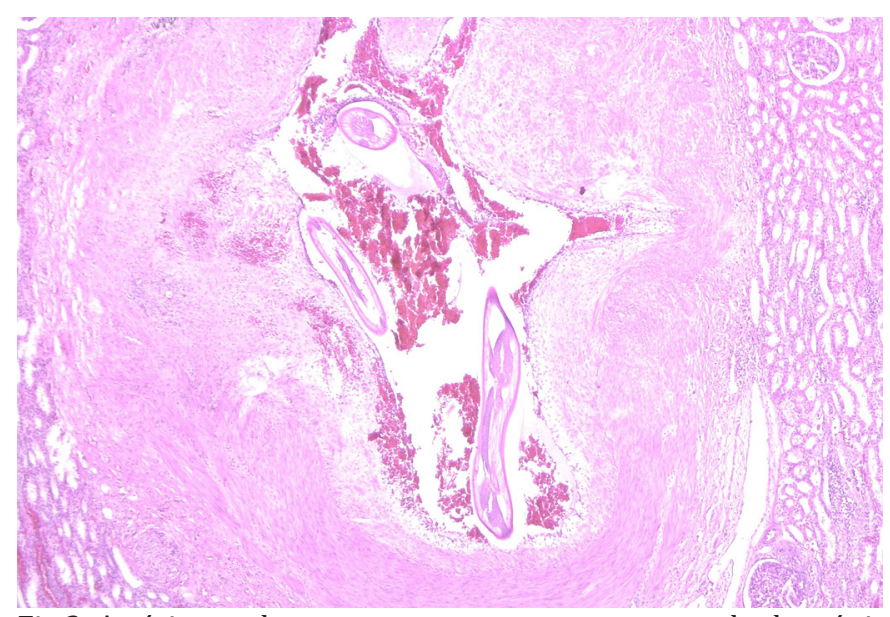

Fig.2. Artéria renal apresenta espessamento acentuado das túnicas íntima e média, três filarídeos adultos consistentes com Cruorifilaria tuberocauda no lúmen e hemorragia multifocal na íntima (Capivara C2). HE, obj.10x.

estavam bastante distorcidas e com espessamento acentuado das túnicas íntima e média que se projetavam para a luz dos vasos (Fig.2). Na túnica íntima, havia proliferação acentuada de células endoteliais e da lâmina conjuntiva subendotelial. Ocasionalmente, a luz arterial estava bastante ocluída e, em alguns casos, havia perda de células endoteliais. A túnica média apresentava áreas multifocais com perda da arquitetura tissular com debris celulares (necrose), algumas vezes associada a infiltrado leve de eosinófilos e macrófagos com raras células gigantes multinucleadas e mineralização moderada. Em algumas artérias, esse infiltrado também afetava a adventícia. Na luz de muitas artérias, foram notados cortes transversais e longitudinais de organismos do Filo Nematoda, Classe Chromadorea (Secernentea), Ordem Spirurida e superfamília Filarioidea. Os parasitos caracterizavam-se por apresentar pseudoceloma, diâmetro variando de 300 a $600 \mu \mathrm{m}$, cutícula externa lisa e fina, musculatura celomiária, cordas laterais pequenas divididas por uma crista cuticular interna e intestino pequeno (Fig.2). Os nematódeos fêmeas possuíam útero grande 


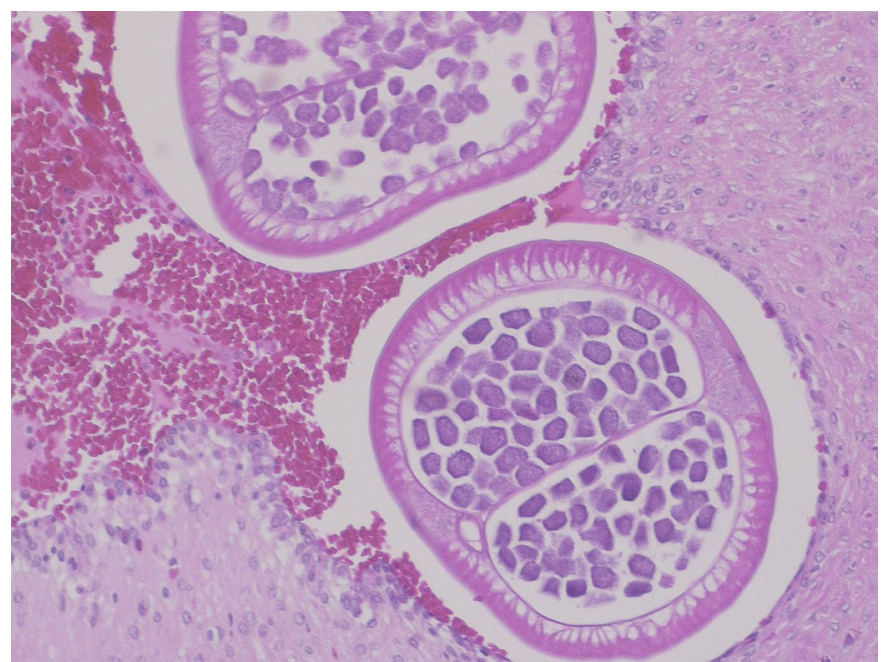

Fig.3. Centro da lesão renal com detalhe de duas fêmeas de filarídeos onde nota-se cutícula externa lisa e delgada, musculatura celomiária, cordas laterais pequenas divididas por uma crista cuticular interna e numerosos ovos variando de 20 a 40 $\mu \mathrm{m}$ de diâmetro (Capivara C1). HE, obj.40x.

preenchido com numerosos ovos, que variavam de 20 a 40 $\mu \mathrm{m}$ de diâmetro (Fig.3). Os parasitos machos continham testículo grande, preenchendo grande parte do pseudoceloma.

Adicionalmente, observou-se nefrite intersticial linfoplasmocítica multifocal leve, glomerulonefrite proliferativa e glomeruloesclerose moderadas. No pulmão, havia algumas áreas aleatórias focais de necrose com infiltrado de eosinófilos.

\section{DISCUSSÃO}

No presente estudo, as capivaras morreram (naturalmente ou por eutanásia) por motivos distintos e, mesmo diante da gravidade das alterações vasculares renais detectadas, as mesmas foram consideradas achados acidentais, em ambos os casos. Os achados histomorfológicos dos parasitas presentes nas lesões renais são compatíveis com o filarídeo Cruorifilaria tuberocauda. Esse parasita é o único filarídeo descrito em capivaras (Eberhard et al. 1976) e as principais lesões ocorrem no rim. Em alguns casos, as lesões renais não são visualizadas macroscopicamente (Vieira et al. 2006). Infartos renais piramidais já foram descritos em áreas adjacentes a vasculite causada por $C$. tuberocauda (Eberhard et al. 1976, Nascimento et al. 2000). Essa lesão não foi observada nos animais do presente estudo. Alguns autores citam que a lesão renal causada pelo $C$. tuberocauda é caracterizada por projeções vilosas e crescentes da parede interna dos vasos para o interior do lúmen, como em uma tentativa de cercar o filarídeo (Morales et al. 1978). Ocasionalmente, essa lesão granulomatosa vascular pode se estender para o parênquima renal (Morales et al. 1978). Além de causar arterite proliferativa nos rins, C. tuberocauda pode causar a mesma lesão em vasos pulmonares e coronarianos de capivaras (Eberhard et al. 1976, Morales et al. 1978). Nos casos do presente trabalho, não foram observadas lesões no coração. No pulmão, notaram-se apenas áreas focais de necrose contendo infiltrado de eosinófilos que podem estar relacionadas à migração de larvas do filarídeo.

Lesões vasculares parasitárias graves como as observadas no presente trabalho podem ocorrer similarmente em cães parasitados por Dirofilaria immitis, Spirocerca lupi e Angyostrongylus vasorum, especialmente na artéria pulmonar e átrio direito cardíaco, na aorta torácica e em vasos pulmonares, respectivamente. Dentre outros nematódeos que podem causar alterações vasculares semelhantes, destacam-se Strongylus vulgaris na artéria mesentérica cranial de equinos, Eleophorapoelie schneideri nas artérias de ruminantes e Onchocerca armillata na aorta de ruminantes e camelídeos (Maxie \& Robinson 2007).

Pouco se conhece sobre a patogenia da infecção por $C$. tuberocauda. Lesões renais, semelhantes às descritas no presente artigo, já foram observadas em porcos selvagens (Sus scrofa) na Colômbia onde ocorrem casos de parasitismo por C. tuberocauda em capivaras (Eberhard et al. 1976, Morales et al. 1978). Esses autores sugerem que os porcos participam da epidemiologia da parasitose na Colômbia como hospedeiros acidentais ou naturais. No Distrito Federal, até o momento, não foram observadas se outras espécies de animais silvestres de vida livre possuem lesões parasitárias semelhantes às detectadas no presente estudo, nem tão pouco se outras espécies poderiam atuar na região como reservatórios ou hospedeiros de $C$. tuberocauda. Futuros estudos são necessários para a determinação dos aspectos epidemiológicos e ecológicos dessa parasitose.

\section{CONCLUSÃO}

Com base nos achados macro e microscópicos, firmou-se o diagnóstico de arterite proliferativa e granulomatosa renal em capivaras associada ao parasitismo por filarídeos consistentes com Cruorifilaria tuberocauda. Pelo conhecimento dos autores, essa parasitose é descrita pela primeira vez em capivaras no Distrito Federal.

\section{REFERÊNCIAS}

Alho C.J.R. \& Rondon N.L 1987. Habitats, population densities, and social structure of capybaras (Hydrochaeris hydrochaeris, Rodentia) in the Pantanal, Brazil. Revta Bras. Zool. 4:139-149.

Campo-Aesen I. \& Planas-Giron G. 1986. Cruorifilaria tuberocauda (Nematoda: Filaroidea) in Venezuelan Capybaras. Medicina Cutanea Ibero-latino-americana 14:109-113.

Corriale M.J., Milano A.M.F., Gómez-Muñoz M.A. \& Herrera E.A. 2011. Prevalence of gastrointestinal parasites in a natural population of capybaras, Hydrochaeris hydrochaeris, in Esteros de Iberá, Argentina. Revta Ibero-Latinoam. Parasitol. 70:189-196.

Costa C.A.F. \& Catto J.B. 1994. Helmintos parasitos de capivaras (Hydrochaeris hydrochaeris) na sub-região de Nhecolândia, Pantanal Sul-Matogrossense. Revta. Bras. Biol. 54:39-48.

Costa D.S., Paula T.A.R., Fonseca C.C. \& Neves M.T.D. 2002. Reprodução de capivaras. Arq. Ciênc. Vet. Zool. Unipar 5:111-118.

Eberhard M.L., Morales G.A. \& Orihel T.C. 1976. Cruorifilaria tuberocaudaGen. et sp. n. (Nematoda: Filarioidea) from the capybara, Hydrochaeris hydrochaeris in Colombia. J. Parasitol. 62:604-607.

Maxie M.G. \& Robinson W.F. 2007. Cardiovascular system, p.1-105. In: 
Maxie M.G. (Ed.), Jubb, Kennedy, and Palmer's Pathology of Domestic Animals. Vol.3. 5th ed. Saunders Elsevier, Edinburgh. 737p.

Morales G.A., Guzman V.H. \& Angel D. 1978. Vascular damage caused by Cruorifilaria tuberocauda in the capybara (Hydrochaeris hydrochaeris). J. Wildl. Dis. 14:15-21.

Nascimento A.A., Bonuti M.R., Tebaldi J.H., Mapeli E.B. \& Arantes I.G. 2000. Natural infections with filarioidea nematodes in Hydrochoerus hydrochaeris in the floodplain of Mato Grosso do Sul, Brazil. Braz. J. Vet. Res. Anim. Sci. 37:105-108.
Ojasti J. 1973. Estudio Biologico de Chiguireo Capibara. Republica de Venezuela. Fondo Nacional de Investigaciones Agropecuarias, Caracas. 181p.

Rodriguez H.C., Brito E.C. \& Perea F.L. 1975. Aspectos fisiologicos y sanitarios del Chiguiro. Cespedesia 4:15-44.

Vieira F.M., Louzada G.L., Lima S.S. \& Bessa E.C.A. 2006. First occurrence of Cruorifilaria tuberocauda Eberhard, Morales et Orihel, 1976 (Spirurida, Filarioidea) in Hydrochaeris hydrochaeris (Linnaeus, 1766) (Rodentia, Hydrochaeridae) in the municipality of Juiz de Fora, Minas Gerais, Brazil. Revta Bras. Zoociênc. 8:83-86. 